\title{
Risk Assessment in Pulmonary Arterial Hypertension According to Right Atrial Pressure and Correlation Between Mean Pulmonary Arterial Pressure with Right Atrial Pressure in Uncorrected Atrial Septal Defect Patients at Dr. Sardjito General Hospital
}

\author{
Dwi Krisnawati*, Dyah Wulan Anggrahini, Anggoro Budi Hartopo, Lucia Kris Dinarti \\ Department of Cardiology and Vascular Medicine, Faculty of Medicine, Universitas Gadjah Mada - \\ Dr. Sardjito Hospital Yogyakarta, Indonesia \\ *correspondence: dr.krisnakitaro@gmail.com
}

\begin{abstract}
Background and aims: Pulmonary arterial hypertension (PAH) sometimes accompanied patient with atrial septal defect (ASD). The 2015 European Society of Cardiology (ESC)/ European Respiratory Society (ERS) pulmonary hypertension $(\mathrm{PH})$ guidelines recommend that risk assessment should be conducted using multiple parameters include a range of clinical, haemodinamic and exercise to evaluate disease progression and patient's response to treatment. Right atrial pressure (RAP) is one of the haemodinamic parameter risk assessment in PAH. The aims of the present study were to investigated whether risk assessment in PAH according to RAP and correlation between mean pulmonary arterial pressure (mPAP) with RAP and in uncorrected ASD patients.

Methods: A cross-sectional study was performed by analyzing right heart catheterization (RHC) data between July 2012 - Mei 2017 and a part of COHARD-PH (Congenital Heart DiseasePulmonary Hypertension) study from 299 adult patients with uncorrected ASD at Dr. Sardjito General Hospital. We used RAP as haemodinamic parameter of risk assessment in PAH. Right atrial pressure and mPAP were measured on RHC. Risk assessment in patients with PAH according to 2015 ESC PH guideline that divided RAP into 3 categories: low risk (RAP $<8 \mathrm{mmHg}$ ), intermediate risk (RAP $8-14 \mathrm{mmHg}$ ) and high risk (RAP $>14 \mathrm{mmHg}$ ). The correlation between RAP and mPAP was assessed using non-parametric Spearman's correlation analysis using SPSS 23.
\end{abstract}

Results: Two hundred-ninety nine consecutive patients with ASD (257 females (86\%) and 42 males $(14 \%)$, mean age $35.94 \pm 12.81$ years) underwent RHC and $209(69.8 \%)$ patients have PAH. The mean RAP and mPAP were $13.09 \pm 5.93 \mathrm{mmHg}$ and $38.78 \pm 20.67 \mathrm{mmHg}$ respectively. Eighteen (8.6\%), $102(48.8 \%$ ) and $89(42.6 \%)$ patients were in the low, intermediate and high risk assessment categories in PAH according to RAP respectively. Mean pulmonary arterial pressure has significant correlation with $\operatorname{RAP}(r=0.340, P=<0.0001)$ in uncorrected ASD patients.

Conclusions: In our study, mostly ASD patients were in intermediate risk followed by high risk and low risk assessment in PAH according to RAP. Mean pulmonary arterial pressure has moderate significant correlation with RAP in uncorrected ASD patients.

Keywords: Atrial septal defect; mean pulmonary arterial pressure; right atrial pressure; risk assessment 